\title{
Serological and Epidemiological Picture of Dengue during the Year 2014: An Exclusive Study of Kutch District, Gujarat, India
}

\author{
Jigar Kiritkumar Gusani*, Hitesh Jayprakash Assudani, Krupali Kothari and A.N. Ghosh \\ Department of Microbiology, Gujarat Adani Institute of Medical Sciences, Bhuj, India \\ *Corresponding author
}

\section{A B S T R A C T}

Keywords

Dengue,

Kutch,

ELISA,

Serological markers, Epidemiology.

Article Info

Accepted:

19 April 2017

Available Online:

10 May 2017
The present study was carried out to find out data on Dengue specific serological markers like NS1 antigen, IgM antibody and epidemiological scenario of Dengue cases in Kutch region during the year 2014. Total 765 blood samples from across the Kutch region were tested by ELISA for NS1 antigen and/or IgM antibody as per the protocols and personal, demographic and clinical details of each patient was recorded. Analysis of this data revealed that age group 21-30 years had maximum dengue cases. Male cases (429) were more than females (336). Even though major chunk of Kutch population leaves in rural areas; overall dengue cases were high in urban areas. Out of 765 cases 331 tested positive for dengue. Amongst all positive cases $181(54.68 \%)$ were tested positive for NS1 antigen which helped in early diagnosis of dengue. Rise of dengue cases started after the month of August and falls down by the end of December 2014 which coincides with post monsoon season.

\section{Introduction}

Dengue is a flavivirus transmitted by Aedes aegypti mosquito. Dengue virus infection has emerged as a notable public health problem in recent decades in term of the mortality and morbidity associated with it (Ukey et al., 2010; WHO, 1997). Dengue is a major problem in many parts of India and large outbreaks are reported from various parts of India and abroad (George et al., 1975; Kaur et al., 1997). The clinical picture of dengue mimics many other viral illnesses, which makes laboratory confirmation very crucial for the patient management. The benefits of early diagnosis of dengue fever go beyond treatment and encase epidemiological efforts. For diagnosis of dengue cases many tests such as virus isolation, RNA, Antigen and antibody detection methods are available (Chakravarti et al., 2006). However virus isolation, RNA detection by PCR, requires well trained staff and dedicated set up which is not feasible in most of the health centers in India. In most of the cases detection of $\operatorname{IgM}$ antibody is used for diagnosis of dengue infection. However time required for appearance of $\operatorname{IgM}$ antibody is approximately 4-6 days (WHO, 1997). Dengue non-structural 1 antigen (NS1) is highly conserved glycoprotein produced in 
both membrane associated and secretary forms is used as a novel biomarker for early diagnosis of dengue infection (Subedi et al., 2014). NS1 antigen detected by ELISA is present in high concentrations in sera of dengue virus infected patients during early clinical phase of disease (Kumarasamy et al., 2007).

Kutch is the largest district of India which has a very special \& diverse ecological system. Literature search suggests that there is scarcity of specific \& exclusive data on prevalence of Dengue in Kutch region (Madhulika Mistry et al., 2015).

The present study was carried out to find out data on Dengue specific serological markers like NS 1 antigen (Ag), IgM antibody and epidemiological scenario of Dengue cases in Kutch region during the year 2014.

\section{Materials and Methods}

This was a prospective \& observational study carried out from January 2014 to December 2014 at Department of Microbiology, Gujarat Adani Institute of Medical Sciences, G K General Hospital, Bhuj.

As a designated sentinel surveillance center by National Vector Borne Disease Control Program (NVBDCP), department is receiving serum samples from Primary \& Community Health centers as well as affiliated private hospitals from all over Kutch for testing and confirmation of suspected Dengue cases. Patients with acute onset of illness, high grade fever, severe headache, backache, musculoskeletal pain or retro-bulbar pain with or without rashes were considered as clinically suspected case of dengue virus infection (Durani et al., 2014).

There was standard laboratory requisition form with Personal, Demographic and clinical details which were recorded in same forms. The date of onset of fever and the date of blood sample collection were also recorded in the data entry form. Onset of fever was taken as Day 0 and accordingly sample age was defined as the gap between the date of onset of fever and the date of collection of blood sample.

Serum was separated and then according to the gap between onset of fever and sample collection type of ELISA test was decided. If the gap was 0 to 4 days then NS1 Ag ELISA was performed and if it was more than 6 days then IgM capture ELISA was performed. For the samples having 5 to 6 days of fever both IgM \& NS1 Ag ELISA were performed. The Dengue IgM capture ELISA kits were supplied from NIV, Pune under NVBDCP and for NS1 Ag detection Platelia dengue NS1 Ag (Bio-Rad) ELISA kits were used. If any of these test came positive by ELISA it was considered as confirmed case and notified to the concerned government authorities.

\section{Results and Discussion}

Total 765 blood samples from across the Kutch region were received at our Institute for serological diagnosis of Dengue fever during the year 2014. Following Figure 1 shows age group wise distribution of clinically suspected and positive dengue cases.

Age group of 21-30 years reported highest number of dengue cases followed by age group of 31-40 years. Figure 2 shows that overall males are more affected then females. Positive cases are also more in males.

From figure 3 it can be observed that $403(52.67 \%)$ cases were from urban areas \& $362(47.32 \%)$ were belonging to rural areas. Positive cases were more from urban areas $(46.65 \%)$ as compared to rural $(39.5 \%)$. 
Table 1 displays Dengue case presented to the hospital after onset of fever and positive cases amongst them. Majority of cases appeared between 3 to 5 days of fever (325 cases) followed by $>5$ days of fever (274 cases). Highest positivity was observed amongst the cases that came to hospital within 2 days of fever.

Table 2 shows Number positive cases detected by different ELISA based tests. More than half $(54.68 \%)$ cases were detected by NS 1 antigen test. While almost $10 \%$ of cases were having both the tests, NS 1 Antigen \& IgM antibody, positive. Figure 4 depicts month wise trend of Dengue cases for the year 2014. Peak rise of cases starts after the month of August and falls down by the end of December 2014. Another interesting finding is there are small peaks of rise in dengue cases noted between the months of April to July.
Out of 765 suspected cases, highest cases were seen in age group of $21-30$ years $(34.64 \%)$ followed by $11-20$ years age group $(22.22 \%)$. Also the positive cases amongst both these group showed similar pattern. These show predilection of dengue infection toward young adults and adolescents. These findings are in accordance to other studies by (Durani et al., 2014 \& Pandey et al., 2012).

Gender distribution of cases revealed that 429 $(56.08 \%)$ cases were males and 336(43.92\%) were females. Amongst the positive cases $202(61.03 \%)$ were males and $129(38.97 \%)$ were females. Overall males were more affected which can be due to various factors like their frequent outdoor activities in this region and less reporting of female cases etc. These findings are similar to other studies by Durani et al., 2014; Piyush tripathi et al., 2008; Nidhi pandey et al., 2012.

Table 1 Dengue cases distribution as per days of fever

\begin{tabular}{|c|c|c|}
\hline $\begin{array}{c}\text { Number of days between } \\
\text { onset of fever and sample } \\
\text { collection }\end{array}$ & $\begin{array}{c}\text { Total numbers of sample } \\
\text { collected / Suspected Cases } \\
(\mathbf{n = 7 6 5})\end{array}$ & Dengue Positive (n=331) \\
\hline $0-2$ days & $166(21.7 \%)$ & $81(48.8 \%)$ \\
\hline $3-5$ days & $325(42.8 \%)$ & $137(42.15 \%)$ \\
\hline$>5$ days & $274(35.8 \%)$ & $113(41.24 \%)$ \\
\hline
\end{tabular}

Table 2 Dengue positive cases by ELISA tests

\begin{tabular}{|l|c|c|}
\hline Test (ELISA Based) & $\begin{array}{c}\text { Numbers of Dengue } \\
\text { positive Cases }(\mathrm{n}=331)\end{array}$ & Percentage \\
\hline NS 1 Antigen & 181 & $54.68 \%$ \\
\hline NS 1 Antigen + IgM Antibody & 32 & $9.67 \%$ \\
\hline IgM Antibody & 118 & $35.65 \%$ \\
\hline
\end{tabular}


Figure 1:Age Group Wise Distribution of Dengue Cases

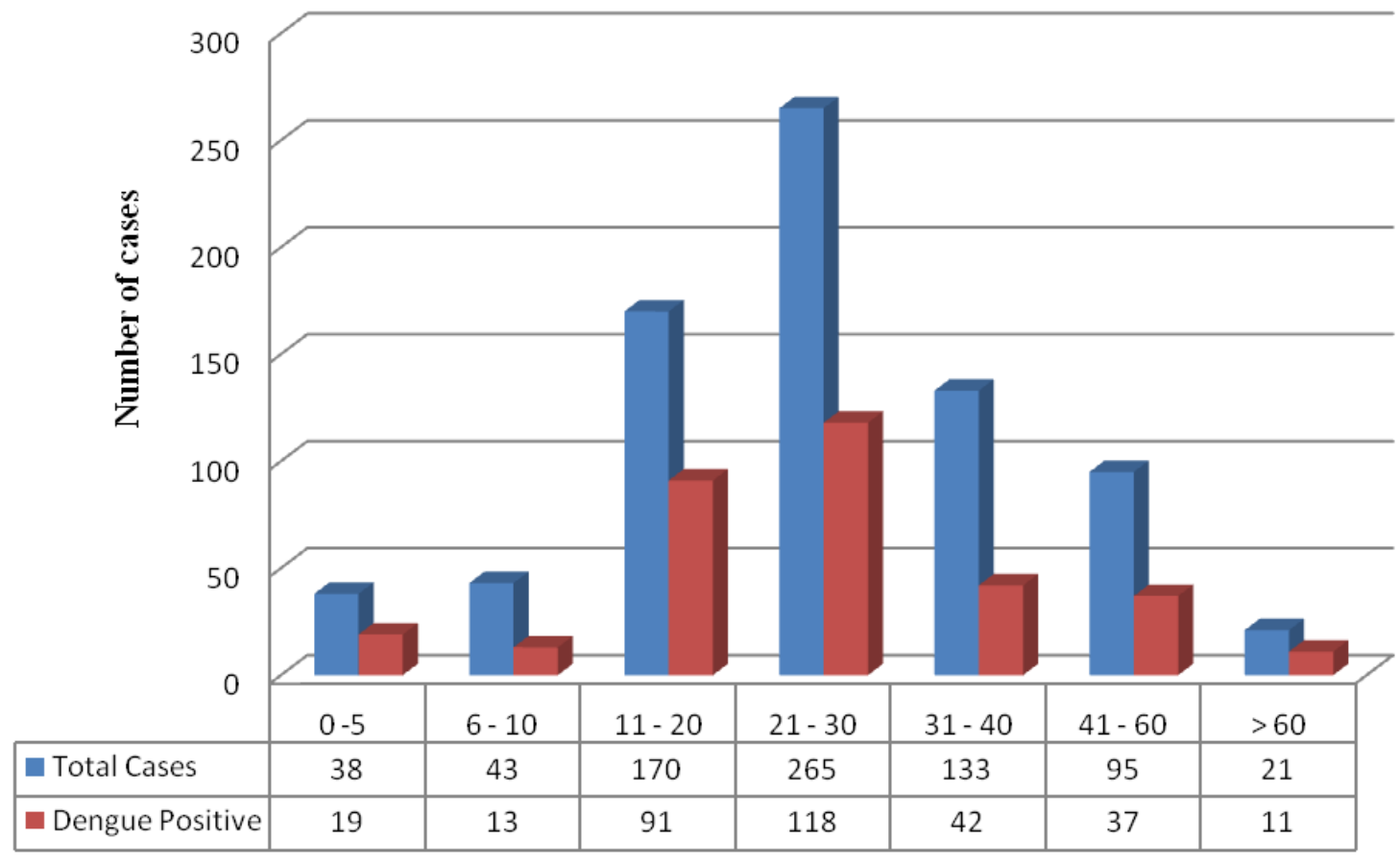

Figure 2: Sex Wise Distribution of Dengue Cases

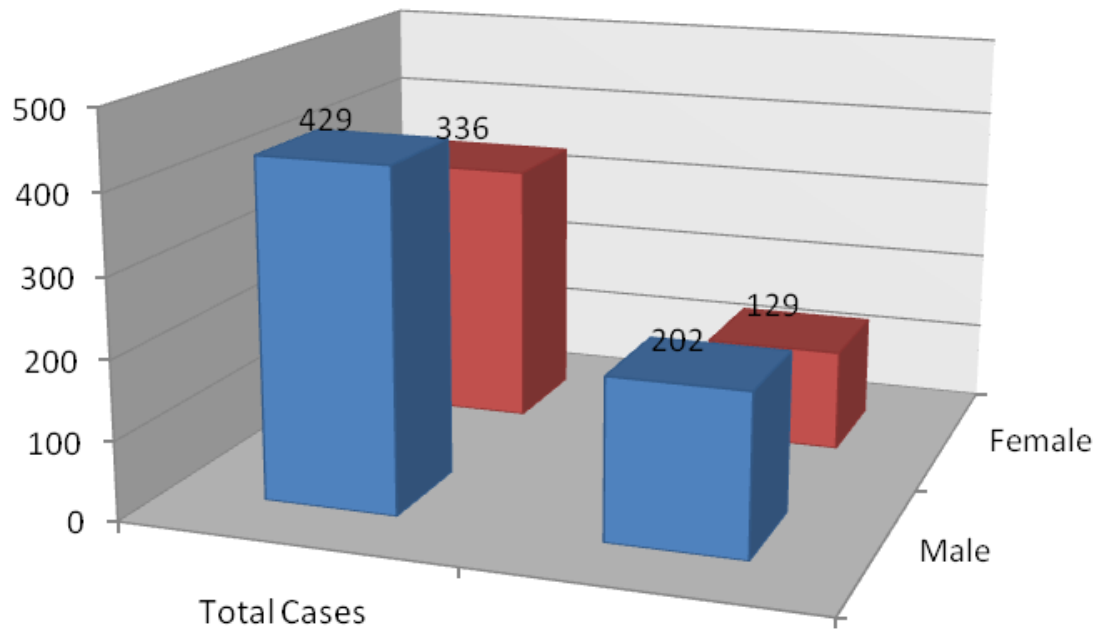

Male

$\square$ Female

Sero Positive Cases 

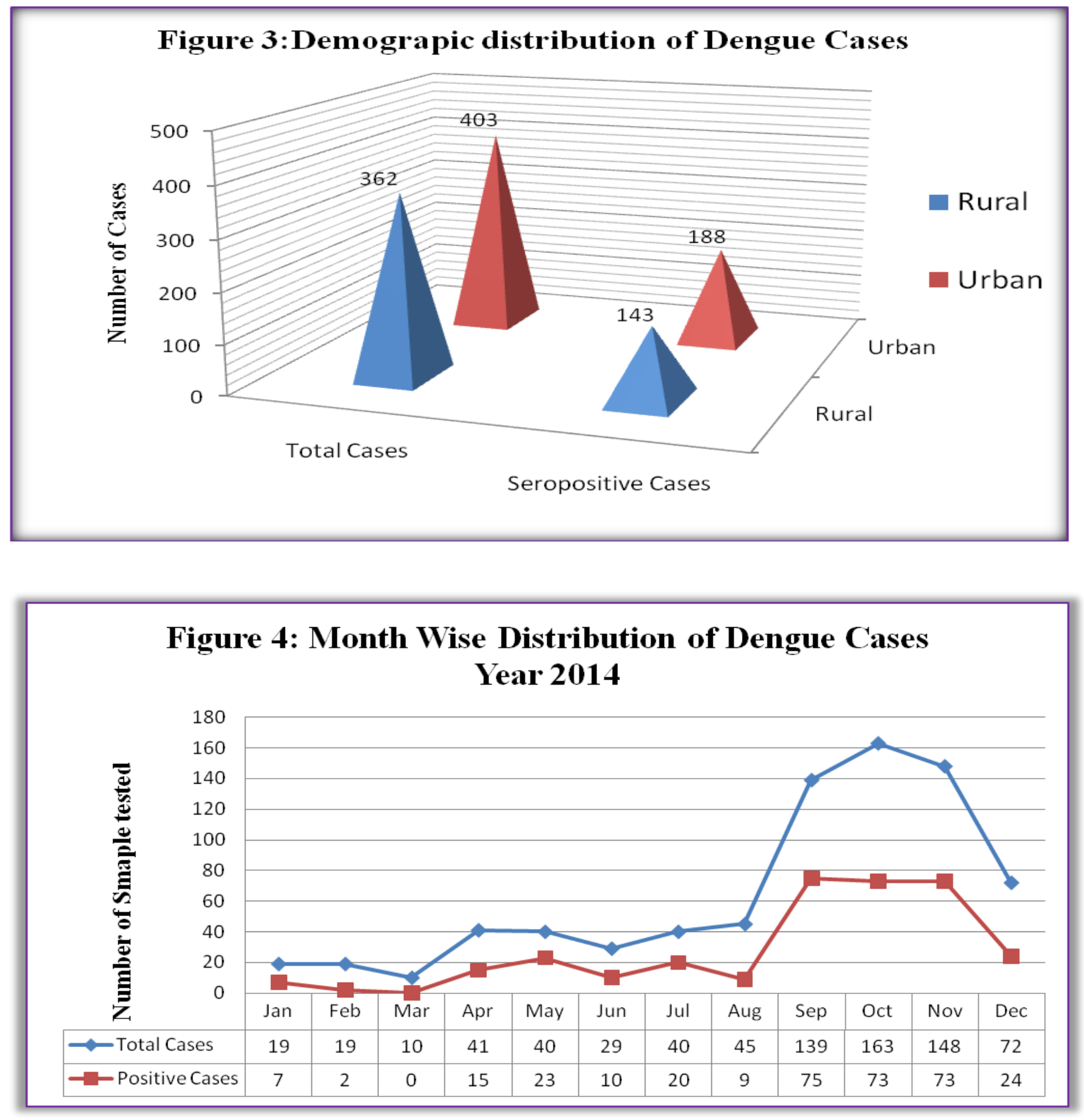

Demographic distribution of Dengue cases in Kutch in 2014 showed that cases from urban areas $(403,52.67 \%)$ were slightly higher than the rural $(362,46.65 \%)$ areas. Although previous studies from Gujarat \& India showed that prevalence of dengue cases were much higher in urban areas; difference here in the present study is much less Madhulika Mistry et al., 2015; Durani et al., 2014; Patankar et al.,
2014; Dutta et al., 2012; Khan et al., 2014. One reason for this can be natural demography of Kutch region where majority of population lives in rural areas as per Census 2011 data (Government of India, 2011 Census).

In this study testing of samples were done as per the age of sample and it was divided into 3 categories based on gap between onset of fever 
and sample collection. As NS1 antigen and IgM antibody levels follow a pattern after the onset of fever; according that the type of test was decided (CDC laboratory guidance for laboratory testing). Maximum number of samples were from 3-5 days of fever (325, $42.48 \%$ ) followed by $>5$ days of fever (274, $35.81 \%)$. However almost half (81, 48.8\%) patients from total 166 patients who came within 2 days of fever were tested positive for Dengue. This could be due to high sensitivity and specificity of Dengue NS 1 antigen ELISA test which was the sole test selected for patients presents early in acute stage of Dengue (Kulkarni et al., 2011; Peeling et al., 2010).

Amongst the positive cases (331) detected in this study, more than half $(181,54.68 \%)$ were alone detected by NS 1 antigen test followed by Ig $\mathrm{M}$ antibody positive cases $(118,35.65 \%)$. In rest of 32 positive cases both NS 1 antigen and Ig M antibody were detected. Various studies have indicated that NS 1 antigen helps in early detection and confirmation of diagnosis (Kulkarni et al., 2011; Datta et al., 2010; Krunal Mehta et al., 2016). When month wise trend was depicted on graph it reinforced the fact that surge in Dengue vector and so the rise in dengue cases occur in Monsoon and post monsoon season (WHO Dengue fever 2nd Edition 1997, Madhulika Mistry et al., 2015; Durani et al., 2014; Piyush tripathi et al., 2008). Rain starts late in Kutch around end of June and lasts till September. In the year 2014 rise in dengue cases occurred from July; peak reached in October and then started declining. Apart from this; there was unusual small peak of cases during the months of April \& May which are hot $\&$ dry months. This requires further epidemiological investigation for search of factors which increased dengue transmission in Non seasonal months. Also there is need of long term studies with analysis of dengue serotypes to know more about the epidemiological, demographic and biological variations of dengue cases in Kutch region. It will tremendously help in taking vector control measures and formulating preventive strategies for Dengue in this region.
It is concluded that this study has just opened the first door for analysis of Dengue in Kutch region. It indicates that young adults, both from rural and urban region, are affected more by Dengue. The high number of positive cases during typical post monsoon season indicates failure in vector control steps and alerts clinicians and epidemiologists to be more vigilant for more such upcoming outbreaks. It also emphasizes role of early detection which can be more accurately done by tests like NS 1 antigen and thus helps in implementation of better clinical and preventive measures for entire population at risk.

\section{Acknowledgment}

We sincerely thank the entire staff of the Department of Microbiology, GAIMS, Bhuj for their support.

\section{References}

Chakravarti A., Kumaria R., Batra V., Verma V. 2006. Improved detection of dengue virus serotypes from serum samples; evaluation of single tube multiplex RTPCR with cell culture. Dengue Bull., 30:133 40.

Datta, S., Wattal, C. 2010. Dengue NS1 antigen detection: A useful tool in early diagnosis of dengue virus infection. Indian J. Med. Microbiol., 28(2): 107-10.

Durani, K., Dund, J., Shingala, H., Sinha, M. 2014. Epidemilogical trend analysis of dengue virus infection in western part of Gujarat. Indian J. Res., 3(6): 146-8.

Dussart, P., Labeau, B., Lagathu, G., Louis, P., Nunes, M.R.T., Rodrigues, S.G., et al. 2006. Evaluation of an enzyme immunoassay for detection of dengue virus NS1Antigen in human serum. Clin. Vaccine Immunol., 13: 118589.

Dutta, P., Khan, S.A., Borah, J., Mahanta, J. 2012. Demographic and clinical features of patients with dengue in Northeastern region of India: a retrospective cross-sectional study during 2009-2011. J. Virol. Microbiol., article ID 786298. doi: 10.5171/2012.786298. 
George, S., Soman, R.S. 1975. Studies on dengue in Bangalore city: Isolation of virus from man and mosquitoes. Indian J. Med. Res., 63: 396-401.

Kaur, H., Prabhakar, H., Mathew, P., Marshalla, R., Arya, M. 1997. Dengue haemorrhagic fever outbreak in October- november 1996 in Ludhiana, Punjab, India. Indian J. Med. Res., 106: 1-3.

Khan, S.A., Dutta, P., Topno, R., Soni, M., Mahanta, J. 2014. Dengue outbreak in a hilly state of Arunachal Pradesh in northeast India. Scientific World J., 584093. doi: $10.1155 / 2014 / 584093$.

Krunal, D., Mehta, et al. 2016. Study of Correlation between Platelet Count and Serological Markers of Dengue Infection with Importance of NS1 Antigen in Western Region of India. National $J$. Laboratory Med., 5 (2): 55.

Kulkarni, R.D., Patil, S.S., Ajantha, G.S., Upadhya, A.K., Kalabhavi, A.S., Subhada, R.M. et al. 2011. Association of platelet count and serological markers of dengue infection - importance of NS1 antigen. Indian J. Med. Microbiol., 29(4): 359-62.

Kumarasamy, V., Wahab, A.H.A., Chua, S.K., Hassan, Z., et al. 2007. Evaluation of a commercial dengue NS1 antigen- capture ELISA for laboratory diagnosis of acute dengue virus infection. J. Virol. Methods, 140: 75-79.

Madhulika Mistry, Yogesh Goswami, Rajesh, K., Chudasama, Dhara Thakkar. 2015. Epidemiological and demographic characteristics of dengue disease at a tertiary care centre in Saurashtra region during the year 2013. J. Vector Borne Dis., 52: p. 299-303

Nidhi Pandey, Rachna Nagar, Shikha Gupta, Omprakash, Danish Khan, Desh Deepak
Singh,Gitika Mishra, Shantanu Prakash, K.P. Singh, Mastan, Singh \& Amita Jain. 2012. Trend of dengue virus infection at Lucknow, north India (2008- 2010): a hospital based study, Indian J. Med. Res., 136, p 862-867.

Pandey, N., Nagar, R., Gupta, S., Omprakash, Khan, D., Singh, D.D., Mishra, G., Prakash, S., Singh, K.P., Singh, M., Jain, A. 2012. Trend of dengue virus infection at Lucknow, north India (2008- 2010): A hospital based study. Indian J. Med. Res., 136: 862-7.

Patankar, M.C., Patel, B.V., Gandhi, V.P., Shah, P.D., Vegad, M.M. 2014. Seroprevalence of dengue in Gujarat, Western India: a study at a tertiary hospital in north India. Int. J. Med. Sci. Public Health, 3(1): 16-8.

Peeling, R.W., Artsob, H., Pelegrino, J.L., Buchy, P., Cardosa, M.J., Devi, S., et al. 2010. Evaluation of diagnostic tests: Dengue. Nat. Rev. Microbiol., 8: S30-37.

Piyush Tripathi, Rashmi Kumar, Sanjeev Tripathi, J.J. Tambe and Vimala Venkatesh. 2008. Descriptive Epidemiology of Dengue Transmission in Uttar Pradesh. Indian Pediatrics, 45: 315-31.

Subedi, D. and Taylor-Robinson, A.W. 2014. Laboratory diagnosis of dengue infection: current techniques and future strategies. Open J. Clin. Diag., 4: 63-70.

Ukey, P.M., Bondade, S.A., Paunipagar, P.V., Power, R.M., Akulwar, S.L. 2010. Study of seroprevalence of dengue fever in central India. Indian J. Community Med., 35(4): 517-9.

World Health Organization. 1997. Dengue haemorrhagic fever: Diagnosis, treatment, prevention and control. 2nd edition. Geneva, Switzerland.

\section{How to cite this article:}

Jigar Kiritkumar Gusani and Ghosh, A.N. 2017. Serological and Epidemiological Picture of Dengue During the Year 2014: An Exclusive Study of Kutch District, Gujarat, India. Int.J.Curr.Microbiol.App.Sci. 6(5): 2100-2106. doi: https://doi.org/10.20546/ijcmas.2017.605.234 